Cátedra del Prof. A Atiziia.

Hospital Luis Calve Matkenna.

\title{
TONSILECTOMIA ENTRE LOS ESCOLARES DE LA CASA NACIONAL DEL NIÑO
}

\section{Por los Dres. HUMBERTO COOPER y OSCAR CORREA}

Desde hace aproximadamente tres décadas, las tonsilas ocupan un lugar destacade en patología humana. Se las considera formaciones importantes, cuya infección puzde ser. la causa tanto de síntomas banales como de graves cuadros generales y procesos a distancia.

El concepto anterior, derivade de la evidencia clínica, he hecho que la tonsilectomía alcance una alta difusión. Ello ha rido especialmente manifiesto entre los países anglo-sajones, en algunos de los cuales, durante un tiempo, se llegó a ia operación preventiva.

Los resultados de la tonsilertomía han sido analizados y expuestos en numerosas ocasiones. El material lo han constituído hasta ahora pacientes de policlínico. A nadie escapa las enormes dificultades de control que envuelve esta circunstancia. Los estudios de este tipo. debido a su índole misms. deben ser ilevados a través de varios años y en condiciones da observación óptimas. A menos que estos requisitos sean satisfechos, sus conclusiones son inevitablemente equívocas. El presente articulo relata los resultados de dicha intervención en una institución cerrada.

Fisiopatologia.

Las tonsilas palatinas scn ćganos linfoideos, de forms de almendra, situados a la entrada de la faringe. Su parénquima, cubierto por una cápsula fibrosa, contiene gran canti- 
dad de nódulos separados por trabéculas a lo largo de las cuales corten vasos sanguíneos, nervios y linfáticos.

La parte más importante de las tonsilas es su porción libre. Ella se presenta revestida por un epitelio estratificado tecamoso, el cual en algunas zonas profundiza y da origen a criptas. Estas, cuyas dimensiones son variables. de $1 \mathrm{~mm}$ a J cm., están comunicadas por finos conductos con la super ficie. En no raras ocasiones, con motivo de inflamaciones crónicas o agudas repetidas, dichas comunicaciones se cierran con la retención consiguiente de material séptico. Se constituyen. en esta forma, pequeños abscesos, cuya evolución es variada. Así, mientras hay algunos que se abren al exterior. que son naturalmente los de curso más benigno, existen muchos que tienden a persistir y aur otros que se extienden por e! tejido tonsilar hasta alcanzar, a veces, las regiones vecinas. Es superfluo recalcar las altas prebabilidades de infección general que existe en cualquiera de estos casos.

Respecto a las funciones de las tonsilas, aunque hay desxuerdo sobre el número e importancia de ellas, todos retonocen su rol como órgano de defensa. El sería especialmente valiosc durante los primeros años. Tal parece desprenterse de estudios histopa-inmunológicos. Se ha logrado, en efecto, comprobar varios hechos que, al mismo tiempo que esclareren este punto, prueban que su mecanismo en tal sentido es muiltip?e. Entre ellos se debe señalar la migración de linfocitos de los nódulos tonsilares a las criptas, la absorción de toxirzss con producción de autoinmunización, el transporte por medio die los linfocitos de gérmenes diversos, atenuados, al tejido linfoldeo donde actuarían como vacunas, etc.

Junto a esta función, con todo, la prircipal. se citan a menudo varias otras. Entre ellas figura la producción de secteciones internas y la formación de éemenios sanguíners. Dado su escaso valor, no haremos sino mencionarlas.

Plan de estudio.

Los resultados de la tonsilectomía no pueden opreciars:, como ya se dijo, en el curso de un lapso breve. Para que un estudio en este sentido sea de importancia, se requiere la existencia de un plazo de observación extenso. El se refiere tanto al tiempo que sigue a la operación como al periodo previo a alla. Tal condición es indispensable dada las caracteristicas de los sintomas y cuadros en relación a la infección tonsilar. 
En el presente trabajo se ba concedido especial atencićn a la circunstancia antes mencionada. Se han incluido. in efecto, sólo casos en quienes posible un control dilatado.

\section{Material.}

Nuestrc estudio comprende 32 casos extraídos dei Servicio Médico-Escolar de la Casa Nacional del Niño. Todo. corresponden a escolares tonsileztomizados desde la creación $a_{c}$ : servicio respectivo en este establecimiento, hace seis años. Ellos fueron seleccionados entre alrededor de 70 operados. 1a mayoría de los cuales debieron ser eliminados por no satisfa(er el plazo de observación exigido, e! que se fijó en orko años cuatro previos a la operación y cuatro posteriores a ella. Un gran porcentaje de éstos eran niños sometidos a la intervención breve tiempo después de su ingreso a la Casa Nacional o durante los últimos tres años, y algunos, pacientes cuvo control se perdió por baber sido retirados.

El grupo de estudio lo constituyen únicamente niños sometidos a tcnsilectomía palatina. Se excluyó a los escolates cperados de vegetaciones adenoídeas como también a aquéllos que fueron objeto de la extirpación combinada por totalizar un percentaje relativamente bajo en el material disponible.

Como control se usó un grupo de escolares no tonsilectomizados, tomados al azar entre la población estudianti! del crtablecimiento. En su obtención no se aplicó otro criterio de cclicción que la inexistencia tanto de tonsilectomía y adenoic mía como de su indicación operatoria. Este grupo lo forman 32 escolares de la misma edad y sexo que los operados, seguidos durante un periodo igual al de éstos. Con excepción de algunos casos que sirvieron de control a los tonsilectomizads: más jóvenes y que como aquéllos, debido a su edad, permanecieron el primer año que abarca el estudio en colocación lamiliar, todos convivieron estrechamente con los cperado? en los diferentes servicios de la institución durante el iemno completc del trabajo.

Con el abjeto de avaluar lor efectos de la tonsilectomix $\because$ aralizó la incidencia de varias afecciones antes y después de la intervención. En el cuadro II aparece la lista de ellos.

Según se aprecia en el cuadro citado, se estudiaron en total seis afecciones. Se excluyeron varias que es frecuent? encontrar en trabajos de este carácter, los de Kaiser y Selkirk y Mitchell especialmente, an atención a la inseguridad de los datos respectivos. En esta categoria figuran manifes- 


\section{CUADRO I}

\begin{tabular}{|c|c|c|c|c|c|c|}
\hline $\begin{array}{l}N . y \\
C, y=\mathrm{C}\end{array}$ & Nombre & $\begin{array}{r}\text { No } \\
\text { fi:ha }\end{array}$ & Se:.o & $\begin{array}{l}E t: \cdot 1 \\
\therefore=10 \cdot \pi\end{array}$ & $\mathrm{T} \cdot \mathrm{cth}=$ of. & $\begin{array}{l}\text { Peso (dest. \% } \\
\text { del stantard) }\end{array}$ \\
\hline
\end{tabular}

A.

D.

\begin{tabular}{|c|c|c|c|c|c|c|c|c|}
\hline 1 & J. Y. & 5079 & $\mathrm{M}$. & 10 & $N(b)$ & 37 & $\begin{array}{r}+7 \% \\
-8 \% 1 \\
\end{array}$ & $\begin{array}{l}+12 \% \\
(+3 \%)\end{array}$ \\
\hline 2. & A. $V$. & 2516 & $1:$ & $11 .-3$ & Ozt. ? & $? 8$ & $\begin{array}{c}0 \\
-2 \%\end{array}$ & $\begin{array}{l}-1 \% \\
(+1 \% i\end{array}$ \\
\hline 3. & C. D. & 2923 & $\mathrm{~F}$ & $10-8$ & Dic. 3 & 38 & $\begin{array}{l}-1 \% \\
(-2 \%) \\
(\cdots-1 / r\end{array}$ & $\begin{array}{c}+3 \% \\
+9 \% \\
\end{array}$ \\
\hline 4. & O. Y. & 1351 & $\Gamma$. & $12-5$ & Atr. 3 & $3 B$ & $\begin{array}{l}-3 \% \\
(+4 \%)\end{array}$ & $\begin{array}{l}-2 \% \\
(+12 \%)\end{array}$ \\
\hline 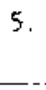 & C. $\mathrm{M}$. & 1900 & $M$ & $10:-1$ & $\mathrm{C}: \mathrm{z}, 3$ & 37 & $-1 \%$ & $\begin{array}{l}+4 \% \\
(+4 \%)\end{array}$ \\
\hline 6. & E. R. & 4290 & $M$ & $?$ & A : 3 & & $\begin{array}{l}-2 \% \\
(-4 \%)\end{array}$ & $\begin{array}{c}0 \\
(-3 \%)\end{array}$ \\
\hline$\therefore$ & C. C. & 2468 &. $\mathrm{M}$ & $11-6$ & $C \therefore, \quad 3$ & 38 & $\begin{array}{l}- \\
(+8 \%)\end{array}$ & $\begin{array}{l}-- \\
-1 \% \\
(+9 \%)\end{array}$ \\
\hline$\varepsilon$. & E. S. & $502 B$ & $\mathrm{M}$. & $9-7$ & $O=\pi, 3$ & 37 & $\begin{array}{l}-1 \% / \% \\
(-5 \%)\end{array}$ & $\begin{array}{l}+6 \% \\
(-2 \%)\end{array}$ \\
\hline 9. & C. $A$. & 1897 & $\mathrm{M}$ & $11 \ldots 9$ & Mir. & 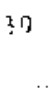 & $\begin{array}{c}+8 \% \\
+6 \% 1\end{array}$ & $\begin{array}{l}+22 \% \\
(-5 \%)\end{array}$ \\
\hline & M. V. & 3372 & $M$ & $10-3$ & $\mathrm{NCv}$ & 38 & $\begin{array}{c}+3 \% \\
(+4 \%) \\
-\end{array}$ & $\begin{array}{l}+6 \% \\
+5 \% \\
\end{array}$ \\
\hline & E. P. & 7731 & $\mathrm{~F}$ & $10-1$ & Jun. 3 & 39 & $\begin{array}{l}-13 \% \\
(-2 \%)\end{array}$ & $\begin{array}{l}-7 \% \\
(-6 \%)\end{array}$ \\
\hline 2. & G. R. & $67+0$ & $\mathrm{M}$ & $8-10$ & Ag. 3 & & $\begin{array}{l}-2 \% \\
-2 \%\end{array}$ & 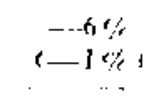 \\
\hline & B. A. & 7076 & $\mathrm{M}$ & $9-8$ & Ag. 3 & & $\begin{array}{c}-1 r_{i} \\
(-11 ; i f\end{array}$ & $\begin{array}{c}+2 \% \\
-12 \% \\
-\end{array}$ \\
\hline & R. B. & 3580 & $\mathrm{E}$ & 9 - & Oor. 3 & 38 & $\begin{array}{l}+8 \% \\
(-.+4 \%)\end{array}$ & $\begin{array}{c}+8 \% \\
10)\end{array}$ \\
\hline 5. & E. $F$ & 7015 & $F$ & $10-2$ & Abr. & 30 & $\begin{array}{r}0 \\
101 \\
-\quad-\quad\end{array}$ & $\begin{array}{l}+3 \% \\
1-2 \%:\end{array}$ \\
\hline 6. & H. M. & 3206 & $M$ & $4-8$ & Dic.? & & $\begin{array}{l}--1] r^{\prime} \\
(-5 \%) \\
--.\end{array}$ & $\begin{array}{c}+3 \% \\
101\end{array}$ \\
\hline . & $Z C$. & 3577 & $1:$ & $0--7$ & Dit: & 37 & $\begin{array}{c}+2 \% \\
1+5 \% 1\end{array}$ & $\begin{array}{l}+12 \% \\
1+10 \%\end{array}$ \\
\hline
\end{tabular}




\begin{tabular}{|c|c|c|c|c|c|c|c|}
\hline \multirow[t]{2}{*}{$\begin{array}{l}\text { N. } \\
\operatorname{caso}\end{array}$} & \multirow[t]{2}{*}{ Nombre } & \multirow[t]{2}{*}{$\begin{array}{l}\text { Nicha } \\
\text { fir }\end{array}$} & \multirow[t]{2}{*}{ Sexo } & \multirow[t]{2}{*}{$\begin{array}{c}\text { Ed-r } \\
\text { añsi-m. }\end{array}$} & \multirow[t]{2}{*}{ l'eche op. } & \multicolumn{2}{|c|}{$\begin{array}{c}\text { Peso (desv. \% } \\
\text { del stantard) }\end{array}$} \\
\hline & & & & & & A. & D. \\
\hline 18. & E. W. & 5419 & $\mathrm{M}$ & $11-10$ & Sер. 38 & $\begin{array}{r}+7 \% \\
(+0 \%)\end{array}$ & $\begin{array}{r}+6 \% \\
+22 \% \\
\end{array}$ \\
\hline 19. & S. C. & 3933 & M. & $9-10$ & Nov. 37 & $\begin{array}{l}-13 \% \\
(+6 \%)\end{array}$ & $\begin{array}{c}-6 \% \\
(+11 \%)\end{array}$ \\
\hline 20. & A. L. & 5050 & $\mathrm{I}$ & $8-5$ & Nov. 37 & $\begin{array}{l}-2 \% \\
(-2 \%)\end{array}$ & $\begin{array}{c}0 \\
(+6 \%) \\
\end{array}$ \\
\hline 21. & H. C. & 5344 & $\mathrm{M}$ & $11-11$ & Sep. 37 & $\begin{array}{r}-4 \% \\
-5 \%)\end{array}$ & $-2 \%$ \\
\hline $2:$ & G. Y. & 5836 & $\mathrm{M}$ & $8-10$ & Otc. 38 & $\begin{array}{c}-2 \% \\
-5 \%) \\
-\infty\end{array}$ & $\begin{array}{r}-5 \% \\
(+3 \%) \\
\end{array}$ \\
\hline 23 & L. M. & 6672 & M. & $9-2$ & Maz. 39 & $\begin{array}{c}+8 \% \\
(+3 \%)\end{array}$ & $\begin{array}{c}+10 \% \\
+13 \%)\end{array}$ \\
\hline 24. & C. J. & 6067 & $\mathrm{M}$ & $8-4$ & Oct. 37 & $\begin{array}{r}+2 \% \\
(-8 \%) \\
\end{array}$ & $\begin{array}{c}+7 \% \\
(-6 \%)\end{array}$ \\
\hline 5. & M. A. & 1542 & $\sqrt{5}$ & I I - - -9 & Jul. 38 & $\begin{array}{r}-5 \% \\
(-7 \%)\end{array}$ & $\begin{array}{r}-2 \% \\
(-6 \%) \\
\end{array}$ \\
\hline 26. & M. V. & $\begin{array}{r}6115 \\
\ldots \ldots\end{array}$ & $\mathrm{M}$. & $11-8$ & Nov. 38 & $\begin{array}{c}0 \\
--8 \%)\end{array}$ & $\begin{array}{c}+2 \% \\
-6 \%\end{array}$ \\
\hline $2 \pi$ & C. P. & 6992 & $\mathrm{M}$ & $9-8$ & $O=t, 37$ & $\begin{array}{c}-9 \% \\
(-4 \%)\end{array}$ & $\begin{array}{r}+1 \% \\
(+11 \%) \\
\end{array}$ \\
\hline E. & S. $\mathbf{G}$. & 6961 & M. & $10-1]$ & Oct. 38 & $\begin{array}{l}-9 \% \\
(0)\end{array}$ & $\begin{array}{c}-4 \% \\
(0)\end{array}$ \\
\hline 29. & A. F. & 3159 & $\mathrm{M}$. & $11-3$ & Sep. 38 & $\begin{array}{r}+4 \% \\
(-13 \%)\end{array}$ & $\begin{array}{c}+5 \% \\
(-17 \%)\end{array}$ \\
\hline 30. & s. 0 & 4607 & $\mathrm{M}$ & $10-2$ & Var 39 & $\begin{array}{r}+2 \% \\
(-4 \%)\end{array}$ & $(\stackrel{+4 \%}{\longrightarrow 3 \%})$ \\
\hline 31. & B. R. & 2254 & $\mathrm{~F}$ & $11-10$ & Mar. 39 & $\begin{array}{c}+4 \% \\
(+6 \%)\end{array}$ & $\begin{array}{r}+9 \% \\
(+16 \%)\end{array}$ \\
\hline 2 & E. M. & 2502 & $\mathrm{~F}$ & $9 \longrightarrow 11$ & Mar. 38 & $\begin{array}{c}-3 \% \\
(+4 \%)\end{array}$ & $\begin{array}{c}+9 \% \\
(+9 \%)\end{array}$ \\
\hline
\end{tabular}

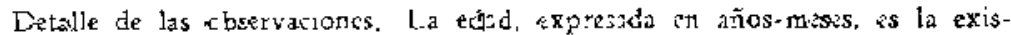
tente en carda niño a la fecha de la operzcion. Las doj últimata columnas con-

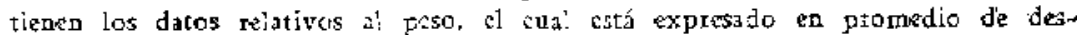

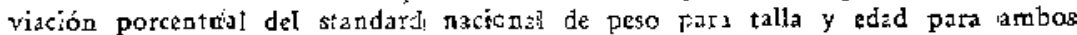
periodos, A. antes de la internación; D. de:fués de ella. Los valorés colocados entre paréntesis corresponden a los casos de control. 
taciones tales como resfríos, cefaleas, etc, que por revestir caracteres atenuados ordinariamente no se declaran. No creemos que pueda concederse gran valor a dichos síntomas, sobre todo en series constituídas a base de clientes de policlínico, debido a la dificultad para obtener por información parental $y$ en forma, a veces, muy tardía, datos que merezcan $f_{e}$.

Respeoto a enfermedades infecto-contagiosas como sarampión, escarlatina y difteria, tampoco las bemos considerado. Ello se debe, con excepción de esta última, tanto a su escasa relación con las formaciones en estudio como a la circunstancia de haberse realizado trabajos inmunológicos pertinentes en ciento número de integrantes de ambos grupos durante los últimos años.

Junto a las características de estudio mencionadas, se consideró de interés precisar la influencia de la operación sobre el estado nutritivo de los niños. Con tal objeto, el peso y estatura de cada niño, obtenidos de sus controles anuales, se compararon con el standard nacional de peso pata talla y edad. La diferencia se expresó en desviación porcentual para ambos períodos. Se eligió el standard citado de peso para talla y edad, y no el de peso para edad, debido al marcado error a que este último expone durante la edad escolar.

En cuanto a clasificación de los casos en normales, obesos y desnutridos se siguió la norma aconsejada por Holt. según la cual debe considerarse normal todo níno cuyo peso no es inferior a $-10 \%$ ni superior a $+15 \%$ del peso normal para talla y edad hasta los 12 años, ni inferior a - $12 \%$ ni mayor a $+20 \%$, sobre dicha edad.

\section{Indicaciones y técnica operatorias.}

A nadie son desconocidas las dificultades que entraña dictaminar sobre el estado de una tonsila. La decisión debe corrientemente ser basada en signos $y$ síntomas que están lejos de ofrecer seguridad. Ni la apariencia del órgano, en efecto, cuya variabilidad es notable, con escasa concordancia, por lo general, entre ella y los hallazgos anátomopatológicos, ni la sintomatologia de la infección tonsilar, de caracteres muy a menudo imprecisos, pueden realmente ser tomados como criterio.

En apoyo de la afirmaciór. anterior creemos oportuno recondar la encuesta realizada hace algunos años por el Dr. Le Mée. Dicho profesor distribuyó entre los principales 
centros médicos del viejo y nuevo continente an cuestionario con lcs puntos más importantes que ordinariamente se discuten al tratar esta materia. Entre los profesionales consultados. ascendentes en total a varias centenas, se contaban pediatras, internistas, cirujanos infantifes y generales, otorinolaringólogos, oftalmólogos y patólogos. Una de las preguntas principales era la relativa al criterio diagnóstico de tonsila séptica, vale decir, en oué casos debía la operación ser indicada. Las respuestas fueron notoriamente dispar:s. Aigrupadas, por orden de mayoria, fueron las siguientes: 1. Eliminación de pus espontáneamente de las criptas o a 'a presión dal pilar anterior; 2. Manifestaciones repetidas do tonsilitis aguda o de absceso peritonsilar; 3. Congestión del pilar anterior; 4. Adenopatía cervical; 5. Presencia o retención de materia caseosa. aun cuando a la presión no se acompañe de pus; 6. Tonsilas "enterradas" o "adherentes", estado derivado de infecciones crónicas o agudas repratidas: 7. Hipentrofia simple tonsilar con aumento de los orificios de las criptas y presencia de pequeñas lesiones úloero-congestivas en sus bordes, y 8 . Examen bacteriológico positivo, esFecialmente si se encuentra el estreptococo.

Hay, como se ye, manifesto desacuerdo en este punto. Sin querer restar su valor a algunos signos, debemos dacir que la opinión de la mayoría de los conocedores del problema, entre ellos autoridades como Epstein y Dean, es desalentadora al respecto, Sostienen que es imposible, en la mayor parte de los casos, clasificar adecuadamente una tonsila sin el examen microscópico.

Como consecuencia de io expueste, se aprecia desdz hace algunos años, especialmente entre los paises de babla inglesa, una nueva actitud frente a las tonsilas. No se opera. en efecto. como antes era muy frecuente en ellos, con un criterio fundado principalmente en el examen local del órgano. sino que se tiende a dar cada vez más importancia al considerar esta posibilidad a la historia clínica dei paciente. I.os datos que ella proporciona, en no raras ocasiones, son de valor mucho mayor que los derivados del examen local.

Nuestro criterio en el curso de este estudio se ciñó a los puntos de vista recién mencionados. Ellos son los mismos que desde hace mucho tiempo se siguen entre nosotros. Formados, en efecto, bajo la influincia serena del profesor Al:jandro del Rio, nunca hemcs compartido los entusiasmo: de algunas näriones. En este sertidọ, nunca se indicó ni m:nes eiecutó una tonsilectomía basados únicamente an el exa. 
men aislado del órgano. Fué la historia del paciente: antecedentes de infección del árbol irspiratorio. frecuencia y graJo de las tonsilitis, existencia de síntomas generales, etc., a ello unido el aspecto de la tonsila y el resultado de su examen detenide, lo que decidió niestra conducta. En general, dadas las pcsibilidades de observación constante de los niños, todas las intervenciones, a excepción de una minoría, se reałizaron después de meses de control.

En lo que se refiere a la operación misma, en su totalidad practicadas por uno de nosctros (H. C.), en la mayor parte de ellas se usó el procedimiento extracapsular, por disección. Aunque de ejecución relativamente lenta es el que. sin duda, ofrece las mayores garantías. No sólo riduce el traumatismo el mínimo y expene en ínfima proporción a complicaciones sino proporciona las mayores seguridades de extirpación total del tejido tonsilar. El método de Sludirs. que usamos en algunos, también excelente, acostumbramos a reservarle para tiños muy peoueños en quienzs el desprendimiento de la cápsula raras veces presenta dificultades.

En cuanto a anestesia. un funto importanti en Pediatría, en todos los casos empleamos la local. Si bien hay citcunstancias en las cuales se ve precisado el cirujano a recurrir a la anesesia general, sea cloro-erérea, con ciclo-propano u otras, como sucede en los niños pequeños, en los de catácter indomable. etc. consideramos la antes mencionada como la dia elección. Además de alejar las posibilidades de complicación y ser mejor tolerada, faciliti en forma clara las maniobras operatorias.

\section{Resultudos.}

Aparecen resumidos en los cuadros II y III. Según se cbserva en el primero de éstos, hubo una marcada dismiuución en la incidencia de la gripe después de la operación. Este efecto es eyidente tanto de la comparación entre los índices respectivos de los operados y controles durance al segundo periodo del estudio como del hecho de su mayor incidencia en el grupo sometido a la internonción en él lapso previo a ésta.

La afección siguiente, laringitis, y con ella las dos que vienen a continuación. bronquitis aguda y neumonía mues. tran, especialmente esta última, un compctamiznte en ciert? forma opuesta al de la gripe. Aun cuando durante el prime: 


\section{CUADRO II}

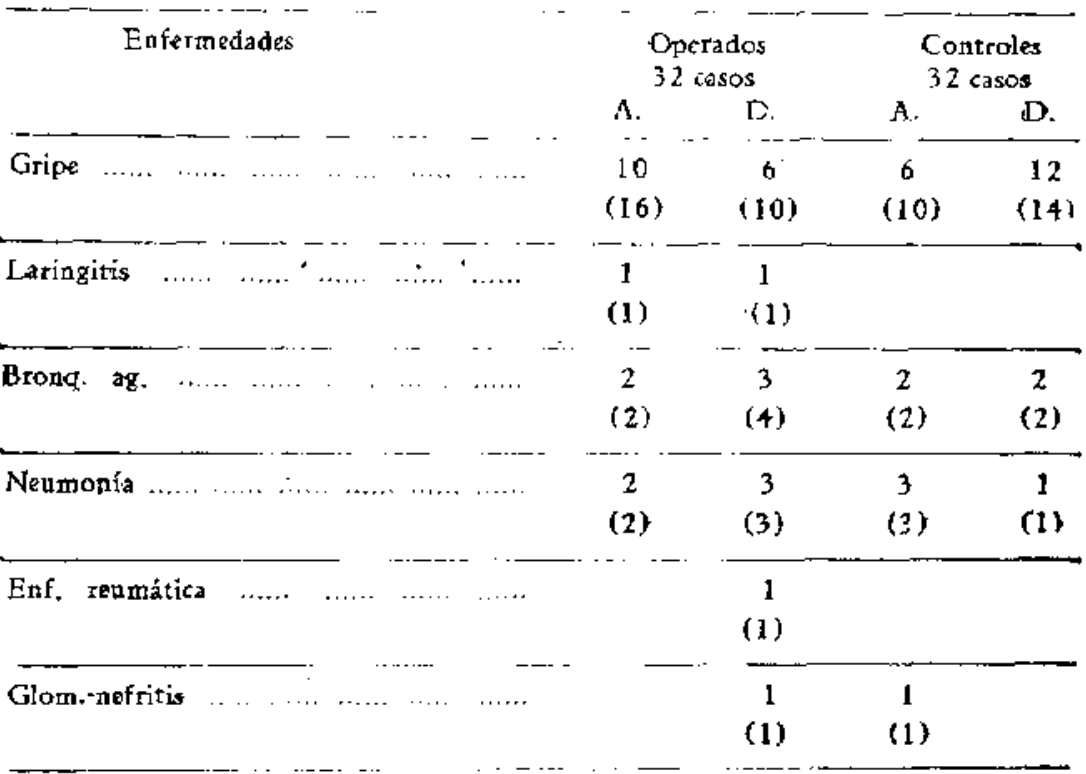

Estudio de 1il morbilidad. Se ha hecho desde el punto de vitata de las seis afecciones anotadas. Con el tbjeto de farihtar la interpretación se dan también los datos correspondientes a los e colare: de control. El cuadro registra tanto la incidencia de una enfermedad por primera vez como sa repetición en an misпro sujeto. Los datos pertenecictité a e te útimo rubro se han colocádo cntre paréntesis. Dado el número idéntico de pacintes y controles, se ha evitado la expresión porcentual, tanto en éste como en el cuadro III. La significación de A. y D. es igual a la indicada en el cuadro I.

\section{OUADRO III}

Pezo

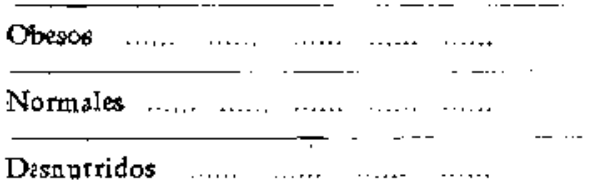

\section{Operados Controles} A. D. A. D.

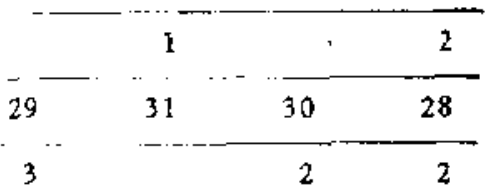

Efacto de la intervención sobre el estado nutritivo. La clasificación se hizo segŕn norma dada a conocer en el texto. El movimiento dz los oasos. lo que en parte se desprende del cuadro, se efectuó en ientido horizontal o ascendente. 
período no parece existir una diferencia digna de mención en su incidencia, ella aumenta, en efecto, después de la operación.

Respecto a los cuadros que siguen, enfermedad reumática y glomérulo-nefritis; sólo nos limitamos a anotar los datos recogidos. Se presentan úricamente por la importancia que se les asigna al considerar esta intervención.

Al lado de las afeccionies mencionadas y no indicada en los cuadros está la tonsilitis aguda. Con excepción de tres casos en quienes se comp̄robó proliferación de tejido linfoideo en la pared posterior de la faringe y que en adelante presentaron infecciones moderadas, los operados no volvieron a padecer este cuadro. Su frecuencia antes de la intervención alcanzaba, por lo menos, a un brote severo al año, en contraste con los casos de control en quienes tal manifestación cra rara.

Concerniente al estudio del estado nutritivo, sus resultados aparecen en el cuadro III. La clasificación se hizo, se gún ya se mencionó, conforme al criterio de Holt. Los revaltados individuales, expresados en desviación porcentual del standard de peso para talla y edad, están expuestos en el cuadro I. De la observación del cuadro III se desprende que la intervención prácticamente no ejerció influencia sobre la nutrición. En efecto, no obstante el alejamiento del standard encontrado en muchos niños, en algunos algo apreciable, tales fluctuaciones quedan casi en su totalidad dentro del margen concedido a los escolares normales durante este período de la vida. El ligero mejoramiento del estado nutritivo acusado por el grupo operado después de la intervención, por supuesto, dentro de los límites de la normalidad, se observa también y más o menos en igual grado en los escolares de control y debe, por lo tanto, set considerado como fisiológico.

\section{Resumen.}

Se estudia el efecto de la tonsilectomía en un grupo de 32 escolares de la Casa Nacional del Niño. Se analiza su morbilidad y estado nutritivo durante cuatro años antes de la operación y durante un periodo igual después de ella. Estos datos se comparan con los obtenidos de un número idén. tico de controles del mismo sexo y edad que los pacientes y en estrecha convivencia con ellos.

Se hace una breve revisión de la fisiopatologia de la tonsilas y de sus indicaciones operatorias. 


\section{BIBLIOGRAFIA}

ABT I.. KAISER A., CASPARIS H. y otros. - Round table dicussica on the tonsil question. Fourth annual neeting of the American Acadeny oi Pediatrics J, of Ped. 7: 137.154 (j:1!o) 1935.

COATES G. y GORDON W. - The tonsit and adenoid problem. J. af Pis $7: 70-78$ (julio) 1935 .

DENZER B. y FELSHIN G. - The pretorsilectomy elinic. J. of P:d. 2Z: 239.249 (febrero) 1943.

KAISER A. - Results of tonsilectomy. I. A. M. A. 95:837.842 (20. 3e s: tiembre) 1930 .

KAISER A. - Significance of the tonsils in the development of the thils. J. A. M. A. 115: 115I-1136 (5 di octubri) 1940.

SELKIRK T. y MITCHELL A. - Evaluation 'of the result: of tonsileren and adenoidectomy, Am, J. Dis. Chicl. 42: 9.41 (julio) 1931. 